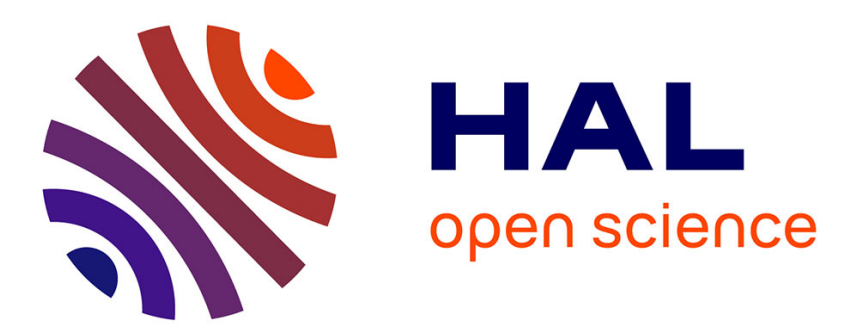

\title{
A multi-body optimization framework with a knee kinematic model including articular contacts and ligaments
}

Nicola Sancisi, Xavier Gasparutto, Vincenzo Parenti-Castelli, Raphaël Dumas

\section{- To cite this version:}

Nicola Sancisi, Xavier Gasparutto, Vincenzo Parenti-Castelli, Raphaël Dumas. A multi-body optimization framework with a knee kinematic model including articular contacts and ligaments. Meccanica, 2017, 52 (3), pp. 695-711. 10.1007/s11012-016-0532-x . hal-01450973

\section{HAL Id: hal-01450973 https://hal.science/hal-01450973}

Submitted on 31 Jan 2017

HAL is a multi-disciplinary open access archive for the deposit and dissemination of scientific research documents, whether they are published or not. The documents may come from teaching and research institutions in France or abroad, or from public or private research centers.
L'archive ouverte pluridisciplinaire HAL, est destinée au dépôt et à la diffusion de documents scientifiques de niveau recherche, publiés ou non, émanant des établissements d'enseignement et de recherche français ou étrangers, des laboratoires publics ou privés. 


\section{A multi-body optimization framework with a knee kinematic model}

\section{including articular contacts and ligaments}

Authors

$5 \quad$ N. Sancisi ${ }^{1}$, X. Gasparutto ${ }^{2}$, V. Parenti-Castelli ${ }^{1}$, R. Dumas ${ }^{2}$

Affiliations

${ }^{1}$ DIN, Health Sciences and Technologies, Interdepartmental Centre for Industrial Research (HSTICIR), Università degli Studi di Bologna, Viale del Risorgimento 2, 40136, Bologna, Italy.

${ }^{2}$ Université de Lyon, F-69622, Lyon, France; Université Claude Bernard Lyon 1, Villeurbanne;

IFSTTAR, UMR_T9406, Laboratoire de Biomécanique et Mécanique des Chocs, F-69675, Bron.

Keywords: Lower limb, gait, soft-tissue artifact, kinematic constraints, penalty-based method, ligament lengthening, contact point position, sensitivity 
Multi-body optimization is one of the methods proposed to reduce the errors due to soft-tissue artifact in gait analysis based on skin markers. This method uses a multi-body kinematic model driven by the marker trajectories. The kinematic models developed so far for the knee joint include a lower pair (such as a hinge or a spherical joint) or more anatomical and physiological representations including articular contacts and the main ligaments. This latter method allows a better representation of the joint constraints of a subject, potentially improving the kinematic and the subsequent static and dynamic analyses, but model definition and mathematical implementation can be more complicated.

This study presents a mathematical framework to implement a kinematic model of the knee featuring articular contacts and ligaments in the multi-body optimization. Two penalty-based methods (minimized and prescribed ligament length variations) consider deformable ligaments and are compared to a further method (zero ligament length variation) featuring isometric ligaments. The multi-body optimization is performed on one gait cycle for five asymptomatic male subjects by means of a lower limb model including the foot, shank, thigh and pelvis. The mean knee kinematics, ligament lengthening and contact point positions are compared over the three methods. The results are also consistent with results from the literature obtained by bone pins or biplane fluoroscopy. Finally, a sensitivity analysis is performed to evaluate how the joint kinematics is affected by the weights used in the penalty-based methods.

The approach is purely kinematic, since the penalty-based framework does not require the solution of the joint static or dynamic analyses and makes it possible to consider ligament deformations without the definition of ligament stiffness that generally cannot be identified through in-vivo measurements. Nevertheless, as far as a knee kinematic model is concerned, particularly in musculoskeletal modeling, this approach seems a good compromise between standard non-physiological kinematic models and complex deformable dynamic models. 


\section{Introduction}

Motion analysis techniques aim at measuring the motion of a subject bones during a considered motor task. A common technique makes use of skin markers, whose positions in space are measured by means of optoelectronic cameras. This technique is not invasive and can be extended to all limbs of the human body, but the relative displacement between markers and bones, known as soft-tissue artifact (STA) [1-3], introduces large errors and inconsistency in this kinematic estimation. Several methods exist to compensate for STA, such as multi-body optimization (MBO) [4-7].

MBO performs a constrained minimization of the distances between the measured skin marker positions and those determined according to a pre-defined kinematic model of the limb. Various kinematic models have been proposed for the joints of the lower limb in this perspective, from simple mechanical joints (hinge, spherical, universal joint) [4-6] to joints with a higher complexity such as parallel mechanisms [7] that introduce more anatomical and physiological degree of freedom (DoF) couplings. Some of the knee parallel mechanisms developed so far [8-12] includes two tibio-femoral contacts and three isometric ligaments: anterior cruciate ligament (ACL), posterior cruciate ligament (PCL) and medial collateral ligament (MCL). They represent an extension in 3D of the classical 2D four-bar mechanism [13-14]. These models proved a high accuracy for subject-specific knee motion replication both in vitro [11] and in vivo [15] and can be easily extended to perform static and dynamic analyses [16-19] that take into account ligament and contact forces separately. However, as they have only one independent DoF, the inter/intra-subject motion variability in the MBO requires subject-specific geometrical identification of the model parameters that need particular attention in invivo measurements [15]. Moreover, the isometric ligament hypothesis cannot represent ligament length variations during flexion, in particular when loads are applied [20-24]. Four deformable ligaments, namely the ACL, PCL, MCL and lateral collateral ligament (LCL), were also introduced in the knee kinematic model based on parallel mechanisms [25]. Two different methods were proposed: in the first one, the ligament length variations were minimized; in the second one, prescribed ligament 
length variations as a function of knee flexion angle were taken as objective of the optimization. Deformable ligaments made it possible to overcome some limitations of isometric ligaments. In general, the use of anatomical knee models based on parallel mechanisms provided encouraging results $[15,25]$ in terms of reduction of errors due to STA, at the expense of a more complicated mathematical implementation with respect to standard mechanical joints. However, the physiological behavior of these knee models in terms of ligament lengthening and contact point positions was not investigated so far.

In this paper, a mathematical framework based on penalty methods is presented to implement deformable ligaments and articular contacts in the MBO. The approach is purely kinematic, since the penalty-based framework does not require the solution of the joint static or dynamic analyses: this aspect reduces the computational burden. Moreover, ligament deformations can be considered without the definition of ligament stiffness, which generally cannot be identified on a subject for in-vivo measurements. For comparison purpose, $\mathrm{MBO}$ is performed also with the same knee kinematic model featuring three isometric ligaments, as previously proposed [7, 25]. The $\mathrm{MBO}$ using this knee kinematic model with zero $\left(\Delta \mathrm{L}_{0}\right)$, minimized $\left(\Delta \mathrm{L}_{\text {min }}\right)$ or prescribed $\left(\Delta \mathrm{L}_{\theta}\right)$ ligament length variations was applied to the gait of five healthy subjects by means of a whole lower limb model and the results of knee kinematics, ligament lengthening and contact point positions were compared. Finally, a sensitivity analysis is performed to evaluate the effect on kinematics of weights used in the penaltybased framework.

85

\section{$2 \quad$ Material \& Method}

\section{$2.1 \quad$ Knee kinematic model}

The knee model used in this study is composed of two sphere-on-plane contacts (representing the two contacts between the femur condyles and the tibia plateaus) and four ligaments, namely the ACL, PCL, MCL and LCL (Fig. 1). The two contacts are rigid and separation is not allowed. The geometry of the model is determined from previous in vitro experimental measurements on a representative 
specimen [11, 26] and is expressed in the femur segment coordinate system (SCS) [27], whose origin is placed at the midpoint between the epicondyles. The tibia SCS is superimposed to the femur SCS at the neutral pose (i.e., static full extension). Details are provided below.

95

\subsubsection{Parallel mechanism}

A parallel mechanism including the two sphere-on-plane contacts and the ACL, PCL, MCL is defined, whose preliminary geometry is obtained from the in vitro measurements $[11,26]$. The geometry of the contact surfaces (i.e., sphere centers and radii, plane positions and orientations) is devised by approximating the femur condyles and tibial plateaus respectively by best-fitting spheres and planes. The geometry of the ligaments (i.e., ligament lengths, origin and insertion coordinates) is identified by finding the isometric fibers, namely the origin and insertion points in each ligament attachment areas that show the minimum distance variation during measured joint natural (i.e., unloaded) motion. These fibers are substituted by links of constant length. Each length is chosen preliminarily as the mean distance between origin and insertion during measured natural motion. This preliminary surface and ligament geometry is then adjusted, so that the mechanism and joint experimental natural motions fit optimally. All geometrical parameters are bounded to keep the final mechanism geometry close to the preliminary estimate [26]. The adjusted sphere centers, plane positions and orientations and ligament origins and insertions are obtained in the corresponding femur and tibia SCSs (Table 1) together with the mechanism sphere radii and ligament lengths $\tilde{d}^{l}(l=1,2$ for medial and lateral condyles and $l=$ 3, 4, 5 for ACL, PCL, MCL, respectively). The whole knee model is obtained by adding the LCL ( $l=$ 6), whose geometry is defined by finding the origin and insertion points in the measured LCL attachment areas with the minimum distance variation during the mechanism motion [28].

\subsubsection{Ligament length variations}

Reference ligament length variations for $\Delta \mathrm{L}_{\min }$ and $\Delta \mathrm{L}_{\theta}$ methods are also defined by the experimental natural motion measured in vitro, so they will be called experimental ligament lengths hereinafter. The adjusted ACL, PCL and MCL fibers in the parallel mechanism model have a constant length during 
the parallel mechanism motion (mechanism ligament lengths $\tilde{d}^{l}$ ). Conversely, the same fibers during the in-vitro natural motion show some small lengthening, since they are not actually perfectly isometric. These lengths, for ACL, PCL, MCL and LCL ( $l=3,4,5,6$, respectively), are obtained as a function of the knee flexion angle $\theta$ (in degree) by computing the distance between the origin and insertion points of the adjusted fibers during measured natural motion. A seventh-order polynomial is used to fit these ligament lengths with a least-square method:

$d^{l}(\theta)=\left(1+a_{1}^{l} \cdot \theta+a_{2}^{l} \cdot \theta^{2}+a_{3}^{l} \cdot \theta^{3}+a_{4}^{l} \cdot \theta^{4}+a_{5}^{l} \cdot \theta^{5}+a_{6}^{l} \cdot \theta^{6}+a_{7}^{l} \cdot \theta^{7}\right) d^{l}(0)$,

where $d^{l}(0)$ are the ligament lengths at the neutral pose $(\theta=0)$.

By construction, since the femur and tibia SCSs are superimposed at the neutral pose, $d^{l}(0)$ can be computed from the distance between the origins and the insertions given in Table 1. It is worth noting that in general $\tilde{d}^{l} \neq d^{l}(0)$, since also the mechanism ligament lengths are adjusted during the parallel mechanism definition (Sect. 2.1.1). The coefficients $a_{1}^{l}$ to $a_{7}^{l}$, are given in Table 2 .

\subsection{Optimization methods}

\subsubsection{Parameters}

MBO is performed by means of a lower limb model including the foot, shank, thigh and pelvis.

Generalized coordinates $\mathbf{Q}_{i}[7,25,29,30]$ are used to represent the pose of each segment $i$. These coordinates consist of two position vectors $\left(\mathbf{r}_{\mathrm{Pi}}\right.$ and $\left.\mathbf{r}_{\mathrm{Di}}\right)$ for $P_{i}$ and $D_{i}$, namely the proximal and distal endpoints respectively, and two unitary direction vectors $\left(\mathbf{u}_{i}\right.$ and $\left.\mathbf{w}_{i}\right)$, representing the directions of two reference axes for the segment:

$$
\mathbf{Q}_{i}=\left[\begin{array}{llll}
\mathbf{u}_{i} & \mathbf{r}_{P_{i}} & \mathbf{r}_{D_{i}} & \mathbf{w}_{i}
\end{array}\right]^{T},
$$

with $i=1,2,3$ and 4 for the foot, shank, thigh and pelvis, respectively.

Parameters in Eq. (2) are designed to stand for anatomical and functional directions representative of the segment and joints anatomy and physiology [30]. Thereby, segment length $\left(L_{i}=\left\|\mathbf{r}_{P_{i}}-\mathbf{r}_{D_{i}}\right\|\right)$, flexion axis of the proximal joint and segment sagittal plane are embedded in those parameters. The 
position of any point of the segment $i$ (both the model-determined skin markers and "virtual markers" standing for the sphere centers, plane positions, ligament origins and insertions) is obtained in the inertial coordinate system (ICS) by a constant interpolation matrix $\mathbf{N}_{i}$. Twelve parameters are used to represent the attitude and position of each segment (Eq. (2)). Consequently, six rigid body constraints are introduced for each segment:

$$
\boldsymbol{\Phi}_{i}^{r}=\left(\begin{array}{c}
\mathbf{u}_{i}^{2}-1 \\
\mathbf{u}_{i}\left(\left(\mathbf{r}_{P_{i}}-\mathbf{r}_{D_{i}}\right)-L_{i} \cos \gamma_{i}\right. \\
\mathbf{u}_{i} \square \mathbf{w}_{i}-\cos \beta_{i} \\
\left(\mathbf{r}_{P_{i}}-\mathbf{r}_{D_{i}}\right)^{2}-L_{i}^{2} \\
\left.\left(\mathbf{r}_{P_{i}}-\mathbf{r}_{D_{i}}\right)\right] \mathbf{w}_{i}-L_{i} \cos \alpha_{i} \\
\mathbf{w}_{i}^{2}-1
\end{array}\right)
$$

with $\alpha_{i}, \beta_{i}, \gamma_{i}$ constant angles of the $\mathrm{i}^{\text {th }}$ segment.

\subsubsection{Constraints}

In the MBO method [7], three types of constraints are needed: the driving constraints $\boldsymbol{\Phi}^{m}$, the kinematic constraints $\boldsymbol{\Phi}^{k}$, and the rigid body constraints $\boldsymbol{\Phi}^{r}$, as mentioned above. The driving constraints represent the distances between the measured and the model-determined skin marker positions, while the kinematic constraints represent the geometrical relationships between the virtual markers, imposed by the kinematic models at the knee, ankle and hip. All constraints are linear or quadratic in the generalized coordinates $\mathbf{Q}_{i}$. The constraints $\boldsymbol{\Phi}^{k}$ are separated into two parts, $\boldsymbol{\Phi}^{k_{1}}$ and $\boldsymbol{\Phi}^{k_{2}} \cdot \boldsymbol{\Phi}^{k_{1}}$ correspond to the knee ligament constraints:

$$
\boldsymbol{\Phi}^{k_{1}}=\left(\begin{array}{l}
\left(\mathbf{N}_{3}^{V_{3}^{3}} \mathbf{Q}_{3}-\mathbf{N}_{2}^{V_{2}^{8}} \mathbf{Q}_{2}\right)^{2}-\left(d^{3}\right)^{2} \\
\left(\mathbf{N}_{3}^{V_{3}^{4}} \mathbf{Q}_{3}-\mathbf{N}_{2}^{V_{2}^{9}} \mathbf{Q}_{2}\right)^{2}-\left(d^{4}\right)^{2} \\
\left(\mathbf{N}_{3}^{V_{3}^{5}} \mathbf{Q}_{3}-\mathbf{N}_{2}^{V_{2}^{10}} \mathbf{Q}_{2}\right)^{2}-\left(d^{5}\right)^{2} \\
\left(\mathbf{N}_{3}^{V_{3}^{6}} \mathbf{Q}_{3}-\mathbf{N}_{2}^{V_{2}^{11}} \mathbf{Q}_{2}\right)^{2}-\left(d^{6}\right)^{2}
\end{array}\right),
$$


with $\mathbf{N}_{i}^{V_{i}{ }^{j}}$ the interpolation matrix for the $j^{\text {th }}$ virtual markers embedded in the $i^{\text {th }}$ segment, and $d^{l}$ the model ligament lengths (Table 1). Specifically, the model ligament lengths $d^{l}$ can be constant (like in $\Delta \mathrm{L}_{0}$ and $\Delta \mathrm{L}_{\min }$ ) or depending on the knee flexion angle $\theta$ (like in $\Delta \mathrm{L}_{\theta}$ ). $\boldsymbol{\Phi}^{k_{2}}$ correspond to the other kinematic constraints of the model: the two sphere-on-plane contacts at the knee, the spherical joint at the hip and the parallel mechanism at the ankle as in [7]. The other constraints, i.e., the driving constraints $\boldsymbol{\Phi}^{m}$ and rigid body constraints $\boldsymbol{\Phi}^{r}$, remain also the same as in [7].

\subsubsection{Ligaments with zero length variation}

Optimization with isometric ligaments is performed using a Lagrange multiplier method. The constrained optimization is formulated as in [7]:

$$
\begin{aligned}
& \min _{\mathbf{Q}} f=\frac{1}{2}\left(\boldsymbol{\Phi}^{m}\right)^{T} \boldsymbol{\Phi}^{m} \\
& \text { subject to }\left\{\begin{array}{l}
\boldsymbol{\Phi}^{k_{1}}=\mathbf{0} \\
\boldsymbol{\Phi}^{k_{2}}=\mathbf{0} \\
\boldsymbol{\Phi}^{r}=\mathbf{0}
\end{array}\right.
\end{aligned}
$$

with $\mathbf{Q}=\left[\begin{array}{llll}\mathbf{Q}_{1} & \mathbf{Q}_{2} & \mathbf{Q}_{3} & \mathbf{Q}_{4}\end{array}\right]^{T}$.

This is equivalent to a zero-search problem when using a Lagrange formulation [6]:

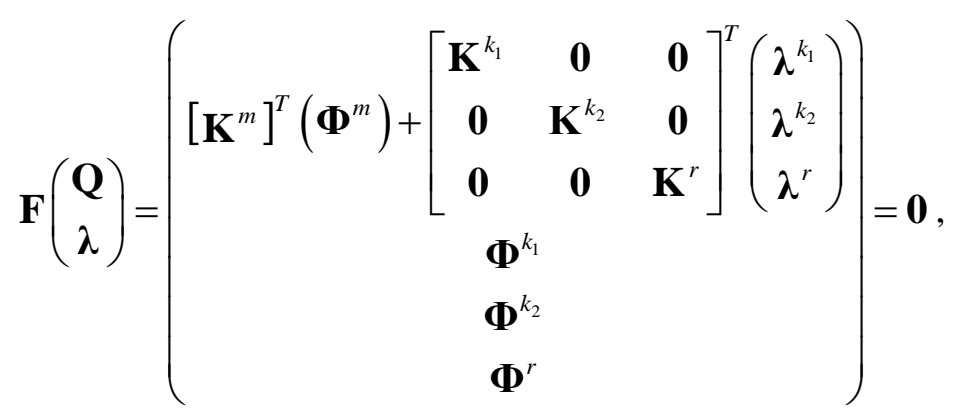

175 with $\mathbf{K}^{m}=\frac{d \Phi^{m}}{d \mathbf{Q}}, \mathbf{K}^{k_{1}}=\frac{d \Phi^{k_{1}}}{d \mathbf{Q}}, \mathbf{K}^{k_{2}}=\frac{d \boldsymbol{\Phi}^{k_{2}}}{d \mathbf{Q}}, \mathbf{K}^{r}=\frac{d \boldsymbol{\Phi}^{r}}{d \mathbf{Q}}$ and with $\lambda^{k_{1}}, \lambda^{k_{2}}, \lambda^{r}$ the Lagrange multipliers associated with the constraints.

In this case, the knee ligament constraints $\boldsymbol{\Phi}^{k_{1}}$ contain only the first three lines (for the ACL, PCL, MCL) of Eq. (4) and the model ligament lengths are constant, set at the mechanism ligament lengths (Table 1): 


\subsubsection{Ligaments with minimized length variation}

Optimization with deformable ligaments is performed as a variation of the previous method with isometric ligaments, and makes use of a penalty-based method. The knee ligament constraints $\boldsymbol{\Phi}^{k_{1}}$ are way:

$$
\begin{aligned}
& \min _{\mathbf{Q}} f=\frac{1}{2}\left(\begin{array}{l}
\boldsymbol{\Phi}^{m} \\
\boldsymbol{\Phi}^{k_{1}}
\end{array}\right)^{T}\left[\begin{array}{cc}
\mathbf{W}^{m} & \mathbf{0} \\
\mathbf{0} & \mathbf{W}^{k_{1}}
\end{array}\right]\left(\begin{array}{l}
\boldsymbol{\Phi}^{m} \\
\boldsymbol{\Phi}^{k_{1}}
\end{array}\right) \\
& \text { subject to }\left\{\begin{array}{l}
\boldsymbol{\Phi}^{k_{2}}=\mathbf{0} \\
\boldsymbol{\Phi}^{r}=\mathbf{0}
\end{array}\right.
\end{aligned}
$$

Consequently, the zero-search problem is modified:

$$
\mathbf{F}\left(\begin{array}{c}
\mathbf{Q} \\
\boldsymbol{\lambda}
\end{array}\right)=\left(\left[\begin{array}{cc}
\mathbf{K}^{m} & \mathbf{0} \\
\mathbf{0} & \mathbf{K}^{k_{1}}
\end{array}\right]^{T}\left[\begin{array}{cc}
\mathbf{W}^{m} & \mathbf{0} \\
\mathbf{0} & \mathbf{W}^{k_{1}}
\end{array}\right]\left(\begin{array}{l}
\boldsymbol{\Phi}^{m} \\
\boldsymbol{\Phi}^{k_{1}}
\end{array}\right)+\left[\begin{array}{cc}
\mathbf{K}^{k_{2}} & \mathbf{0} \\
\mathbf{0} & \mathbf{K}^{r}
\end{array}\right]^{T}\left(\begin{array}{l}
\boldsymbol{\lambda}^{k_{2}} \\
\boldsymbol{\lambda}^{r}
\end{array}\right)\right)=\mathbf{0},
$$

with $\mathbf{W}^{m}$ and $\mathbf{W}^{k_{1}}$ two diagonal weight matrices associated to the driving constraints and the knee ligament constraints respectively.

In a STA compensation perspective, the ligament weights should be much higher than the skin marker weights, but without impeding the optimization convergence. This means that the model kinematics is prioritized with respect to the marker trajectories: ligament lengthening is allowed, but the model hypotheses (i.e., the ligaments remain almost isometric during motion) is preserved. Matrix $\mathbf{W}^{m}$ is set to identity while the four weights used in the diagonal matrix $\mathbf{W}^{k_{1}}$ are given in Table 3. The choice of the weights for each ligament is based qualitatively on the experimental ligament length $d^{l}(\theta)$ and on the literature [20-22]: ligaments with a smaller length variation during knee flexion have higher weights. 
In this case, the knee ligament constraints $\boldsymbol{\Phi}^{k_{1}}$ contain all four lines (for the ACL, PCL, MCL, LCL) of Eq. (4). In order to minimize the ligament length variations, the objective ligament lengths in the model are constant, set at the mean value of Eq. (1) for all the flexion angles $\theta$ during gait:

$d^{l}=\bar{d}^{l}(\theta)$

\subsubsection{Ligaments with prescribed length variation}

Optimization in this case is still performed using the same penalty-based method as presented above. Thus, the same Eqs. (8)-(9) are used, where the knee ligament constraints $\boldsymbol{\Phi}^{k_{1}}$ contain all four lines of Eq. (4) also in this case. The main difference is that, to target the prescribed ligament length variations as a function of the knee flexion, the objective ligament length in the model is variable in this case, set at the values of Eq. (1) for each flexion angle $\theta$ during gait:

$d^{l}=d^{l}(\theta)$

Since the experimental length variation is prescribed, the same weights are used for all ligaments in matrix $\mathbf{W}^{k_{1}}$ (Table 3). Like in the previous method, these weights are much higher than the skinmarker weights to make the model constraints effective and thus to allow STA compensation.

\subsubsection{Initial guess, geometrical parameters and solution}

The initial guess of $\mathbf{Q}$ for $\Delta \mathrm{L}_{0}$ corresponds to the endpoints and directions ( $\left.\mathbf{r}_{\mathrm{Pi}}, \mathbf{r}_{\mathrm{Di}}, \mathbf{u}_{i}, \mathbf{w}_{i}\right)$ computed using the skin markers $[7,30]$. The initial guess for $\Delta \mathrm{L}_{\min }$ and $\Delta \mathrm{L}_{\theta}$ is the optimal solution of $\Delta \mathrm{L}_{0}$. The knee flexion angle $\theta$ computed with this optimal solution is used to calculate the experimental 220 ligament length $d^{l}(\theta)$ and its mean value $\bar{d}^{l}(\theta)$.

As for the geometrical parameters of the lower limb model, they are computed from the aforementioned initial guess of $\mathbf{Q}$. The parameters involved in the rigid body constraints are computed at each sampled instant of time $k$ and averaged: 


$$
\begin{aligned}
& L_{i}=\frac{1}{n} \sum_{k=1}^{n} \sqrt{\left(\mathbf{r}_{P_{i}}(k)-\mathbf{r}_{D_{i}}(k)\right)^{2}} \\
& \alpha_{i}=\frac{1}{n} \sum_{k=1}^{n} \cos ^{-1}\left(\frac{\left(\mathbf{r}_{P_{i}}(k)-\mathbf{r}_{D_{i}}(k)\right) \square \mathbf{w}_{i}(k)}{L_{i}(k)}\right) \\
& \beta_{i}=\frac{1}{n} \sum_{k=1}^{n} \cos ^{-1}\left(\mathbf{u}_{i}(k) \square \mathbf{w}_{i}(k)\right) \\
& \gamma_{i}=\frac{1}{n} \sum_{k=1}^{n} \cos ^{-1}\left(\frac{\mathbf{u}_{i}(k) \llbracket\left(\mathbf{r}_{P_{i}}(k)-\mathbf{r}_{D_{i}}(k)\right)}{L_{i}(k)}\right)
\end{aligned}
$$

Similarly, the reference positions of the skin markers embedded in the relevant segments are computed from the initial guess of $\mathbf{Q}$ at each sampled instant of time $k$ and averaged. The reference position of the $j^{\text {th }}$ skin markers of the $i^{\text {th }}$ segment is expressed as the coordinates $\left(n_{i}^{M_{i}^{j}}\right)_{u},\left(n_{i}^{M_{i}^{j}}\right)_{v}$ and $\left(n_{i}^{M_{i}^{j}}\right)_{w}$ in the non-orthogonal basis $(\mathbf{u}_{i}, \underbrace{\mathbf{r}_{P_{i}}-\mathbf{r}_{D_{i}}}_{\mathbf{v}_{i}}, \mathbf{w}_{i})$, obtained by a non-orthogonal projection using the marker position $\mathbf{r}_{M_{i}^{j}}$ in the ICS:

$\left(n_{i}^{M_{i}^{j}}\right)_{u}=\frac{1}{n} \sum_{k=1}^{n} \frac{\left.\left(\mathbf{v}_{i}(k) \times \mathbf{w}_{i}(k)\right)\right)\left(\mathbf{r}_{M_{i}^{j}}(k)-\mathbf{r}_{P_{i}}(k)\right)}{\left(\mathbf{u}_{i}(k) \times \mathbf{v}_{i}(k)\right) \square \mathbf{w}_{i}(k)}$

$\left(n_{i}^{M_{i}^{j}}\right)_{v}=\frac{1}{n} \sum_{k=1}^{n} \frac{\left(\mathbf{w}_{i}(k) \times \mathbf{u}_{i}(k)\right)\left[\left(\mathbf{r}_{M_{i}^{j}}(k)-\mathbf{r}_{P_{i}}(k)\right)\right.}{\left(\mathbf{u}_{i}(k) \times \mathbf{v}_{i}(k)\right)\left[\mathbf{w}_{i}(k)\right.}$

$\left(n_{i}^{M_{i}^{j}}\right)_{w}=\frac{1}{n} \sum_{k=1}^{n} \frac{\left(\mathbf{u}_{i}(k) \times \mathbf{v}_{i}(k)\right)\left(\mathbf{r}_{M_{i}^{j}}(k)-\mathbf{r}_{P_{i}}(k)\right)}{\left(\mathbf{u}_{i}(k) \times \mathbf{v}_{i}(k)\right)\left[\mathbf{w}_{i}(k)\right.}$

Moreover, the coordinates $\left(n_{i}^{V_{i}^{j}}\right)_{u},\left(n_{i}^{V_{i}^{j}}\right)_{v}$ and $\left(n_{i}^{V_{i}^{j}}\right)_{w}$ of the $j^{\text {th }}$ virtual markers of the $i^{\text {th }}$ segment is obtained from the knee model geometrical parameters (Table 1) using the marker position $\mathbf{r}_{V_{i}^{j}}$ in the ICS:

$$
\left(\begin{array}{l}
\left(n_{i}^{V_{i}^{j}}\right)_{u} \\
\left(n_{i}^{V_{i}^{j}}\right)_{v} \\
\left(n_{i}^{V_{i}^{j}}\right)_{w}
\end{array}\right)=\left[\mathbf{B}_{i}\right]^{-1} \mathbf{r}_{V_{i}^{j}}^{s}
$$


235 with $\mathbf{B}_{i}=\left[\begin{array}{ccc}1 & L_{i} \cos \gamma_{i} & \cos \beta_{i} \\ 0 & L_{i} \sin \gamma_{i} & \frac{\cos \alpha_{i}-\cos \beta_{i} \cos \gamma_{i}}{\sin \gamma_{i}} \\ 0 & 0 & \sqrt{1-\left(\cos \beta_{i}\right)^{2}-\left(\frac{\cos \alpha_{i}-\cos \beta_{i} \cos \gamma_{i}}{\sin \gamma_{i}}\right)^{2}}\end{array}\right]$

However, for the thigh segment $(i=3),-1$ was added to $\left(n_{i}^{V_{i}^{j}}\right)_{v}$ because, differently from the other segments, the origin of the femur SCS was coincident with endpoint $D_{3}$ instead of $P_{3}$ in the knee model geometrical parameters (Table 1). The coordinates of the skin markers $\left(n_{i}^{M_{i}^{j}}\right)_{u},\left(n_{i}^{M_{i}^{j}}\right)_{v}$ and $\left(n_{i}^{M_{i}^{j}}\right)_{w}$ and virtual markers $\left(n_{i}^{V_{i}^{j}}\right)_{u},\left(n_{i}^{V_{i}^{j}}\right)_{v}$ and $\left(n_{i}^{V_{i}^{j}}\right)_{w}$ allow the computation of

240 the interpolation matrices $\mathbf{N}_{i}^{M_{i}^{j}}$ and $\mathbf{N}_{i}^{V_{i}^{j}}$ involved in the driving constraints $\boldsymbol{\Phi}^{m}$ and kinematic constraints $\boldsymbol{\Phi}^{k}$, respectively.

The zero-search problems Eqs. (6), (9) are then solved by a Gauss-Newton algorithm with specified analytical gradient using Matlab R2012a. The convergence was stopped when $\|\mathbf{F}\|<1 e^{-12}$.

\section{$245 \quad 2.3 \quad$ Knee kinematics, ligament length and contact point computation}

The optimized knee joint kinematics is directly computed from the generalized coordinates $\mathbf{Q}_{i}$ [17]: the femur and tibia SCSs are deduced from $\mathbf{Q}_{2}$ and $\mathbf{Q}_{3}$ and the knee joint angles are computed from the joint coordinate system (JCS) according to ISB recommendation [27]. Specifically, the first axis, $\mathbf{e}_{1}$, of knee JCS is $\mathbf{w}_{3}$ and the third axis, $\mathbf{e}_{3}$, is $\mathbf{v}_{2}=\frac{\mathbf{r}_{P_{2}}-\mathbf{r}_{D_{2}}}{L_{2}}$. The displacement of the tibia relative to the

femur is computed as the non-orthonormal projection of the vector from point $D_{3}$ to $P_{2}$ on the axes of the JCS $\left(\mathbf{e}_{1}, \mathbf{e}_{2}, \mathbf{e}_{3}\right)$ [27].

The ligament lengths after MBO are computed from $\mathbf{Q}_{2}$ and $\mathbf{Q}_{3}$ as the distance between the virtual markers standing for the origins and insertions using the corresponding interpolation matrices $\mathbf{N}_{i}^{V_{i}{ }^{j}}$, 
similarly to Eq. (4). The ligament lengthening is represented as a per cent value of the ligament length

at the neutral pose $d^{l}(0)$. The contact point positions are also computed from $\mathbf{Q}_{2}$ and $\mathbf{Q}_{3}$ as the projection of the virtual markers standing for the condyle centers onto the tibia plateau planes. The contact point positions then are plotted in the axial plane of the tibia SCS.

\subsection{Sensitivity Analysis}

\subsubsection{Weights between driving and knee ligament constraints}

To test the sensitivity of the model to the weights between the driving and the ligament constraints, the prescribed ligament length method was chosen, as the same weight was assigned to each ligament in this method. The weight range for the ligaments was chosen from 1 to $2 \mathrm{e} 4$, namely two times the maximal weight used in the model, while the weight of the driving constraints remained 1. Four hundred simulations where performed for each subjects with a uniform distribution of weights.

\subsubsection{Relative weights between ligament constraints}

The sensitivity analysis of the model to the values of the weight matrix $\mathbf{W}^{k_{1}}$ was performed with the minimized length variation method, as the ligament weights are different in this method. Every combination of the four weights used in the model (i.e. 1e $0,1 \mathrm{e} 2,1 \mathrm{e} 3,1 \mathrm{e} 4)$ for the four ligaments was tested, leading to a total of 256 simulations per subject. To get insights on the influence of each ligament on this analysis, four groups of combinations were defined where one of the four ligaments (that thus identifies the group) had maximal weight and the other three ligaments had any lower weight. Each group contains 27 combinations. The maximal standard deviation for each DoF and each group was computed for each subject. Finally, these maximal standard deviations were averaged over the 5 subjects. We inferred that a lower standard deviation for a DoF and a group is associated with a greater kinematic constraint of the ligament corresponding to that group on this DoF.

\subsection{Application to walking analysis}


The MBO is applied to the data of the same five healthy male subjects as in [7] (age: 28.8 \pm 4.8 years; height: $1.74 \pm 0.09 \mathrm{~m}$; mass: $76.5 \pm 13.5 \mathrm{~kg}$ ). The trajectories of twenty-two skin markers on the right lower limb are recorded at $100 \mathrm{~Hz}$ during one gait cycle. The mean optimized knee kinematics, ligament lengths and contact point positions on the five subjects computed with the different methods $\left(\Delta \mathrm{L}_{0}, \Delta \mathrm{L}_{\min }\right.$ or $\left.\Delta \mathrm{L}_{\theta}\right)$ are compared.

\section{$3 \quad$ Results}

285 The mathematical framework proved to be robust and fast, both with isometric and deformable ligaments. Indeed, each MBO method required seconds $(<5 \mathrm{~s})$ on a standard PC (CPU $2.8 \mathrm{GHz}, 2 \mathrm{~GB}$ RAM) to process each gait cycle (about 130 sampled instants of time) and to find the optimal solution for each frame. No particular numerical problems or instabilities were noticed during computations. The kinematic results relative to the five gait cycles (Figs. 2-3) show that the three methods obtain a similar motion. However, there are actually some differences between the curves representing joint angles and displacements, both in terms of pattern and range.

Concerning the joint angles (Fig. 2), while flexion-extension curves do not vary among methods, larger differences can be noticed for the joint internal-external rotation. In particular, all three methods point out a knee internal rotation during gait, with a similar maximum range (peak value about $16^{\circ}$ ), but the overall patterns are different in particular in terms of timing. Indeed, the curve obtained with $\Delta \mathrm{L}_{0}$ is strictly coupled to the flexion angle: the two peaks match the corresponding flexion peaks (respectively at $15 \%$ and $75 \%$ of the gait cycle). A similar behavior is obtained by $\Delta \mathrm{L}_{\theta}$, but the second peak is anticipated to about $55 \%$ of the gait cycle, close to the toe-off. The curve obtained by $\Delta \mathrm{L}_{\text {min }}$ shows a similar behavior, but the first peak can be barely noticed and the internal rotation increases almost monotonically until the second peak at $55 \%$ of the gait cycle.

As for the joint displacements (Fig. 3), the patterns are similar among all methods, but ranges are different. In particular, displacements obtained by $\Delta \mathrm{L}_{0}$ have a smaller magnitude than those obtained by other methods during the whole gait cycle. Conversely, $\Delta \mathrm{L}_{\min }$ produces the largest displacements, 
with peaks of $8 \mathrm{~mm}, 7 \mathrm{~mm}, 3 \mathrm{~mm}$ of medial, anterior, distal displacement respectively. All these peaks are correlated with the knee flexion peak during the swing phase at $75 \%$ of the gait cycle.

Concerning ligament lengthening (Fig. 4), no change in ligament length is observed with $\Delta \mathrm{L}_{0}$, as expected. However, as previously noted, this ligament length is not coincident with the length at the neutral pose and it is close to the mean length approximating the experimental curves (i.e., $\bar{d}^{l}(\theta)$ ). All methods remain close (less than $3 \%$ of root mean square difference) to the experimental ligament lengths (i.e., $d^{l}(\theta)$ ) (Table 4). The results of $\Delta \mathrm{L}_{\theta}$ are closer to the experimental ligament length than $\Delta \mathrm{L}_{\text {min }}$ for the ACL, MCL and LCL (Table 4) but not for the PCL. The only large difference between the curves is observed for the ACL lengthening, where $\Delta \mathrm{L}_{\min }$ obtains a lengthening peak of $10 \%$ at $75 \%$ of gait cycle, corresponding to the maximal knee flexion.

As for the contact point positions (Fig. 5), all methods yield a posterior translation, noteworthy of a same amount for the lateral condyle. However, this posterior translation is very limited for the medial condyle in $\Delta \mathrm{L}_{0}$ and is coupled with a lateral translation in $\Delta \mathrm{L}_{\min }$ and $\Delta \mathrm{L}_{\theta}$.

Concerning the sensitivity analysis of the model to the weights between the driving and the ligament constraints, Fig. 6 presents all knee kinematics obtained by every considered weight for one typical subject and Fig. 8 presents the ligament lengthening obtained by every considered weight for the same subject. The flexion-extension and proximal-distal displacements are not sensitive to these weights. For adduction-abduction, lateral-medial displacement and anterior-posterior displacement, small ligament weights allow wide ranges and higher variations in the estimated DoFs. Above a weight of 3000 , depending on the subjects, the estimations of these DoFs have more consistent patterns and range. Indeed, the maximal variation of these DoFs between the results with different weigths is reduced on average on the 5 subjects from $2.9 \mathrm{deg}$ to $0.5 \mathrm{deg}$ for adduction-abduction and from 6.7 $\mathrm{mm}$ and $17.3 \mathrm{~mm}$ to $2.1 \mathrm{~mm}$ and $0.6 \mathrm{~mm}$ for the lateral-medial displacement and anterior-posterior displacement respectively. The same effect is observed with ligament lengthening (Fig. 8), above a weight of 3000, the maximal variation between the results obtained with optimizations with different weights is reduced on average for the 5 subjects from $30.7 \%$ to $1.7 \%$ for the ACL, from $20.7 \%$ to 
internal-external rotation, the increase in ligament weight is responsible for a continuous shift of the knee rotation toward the results of $\Delta \mathrm{L}_{0}$ during the stance phase. The most important changes occur during the single stance phase and the late swing with variations reaching 10 degrees.

Concerning the sensitivity study of the relative ligament weights between ligament constraints, Table 5 presents the results for each group, Fig. 7 presents the variations of the DoFs for all considered weight combinations and for the same subject of Fig. 6 and Fig.9 presents the variations of the ligament length for all considered weight combinations for the same subject of Fig.6. Although the mean standard deviations remain small, Fig. 7 shows that different combinations can lead to differences in patterns up to 4 degrees for adduction-abduction, 5 degrees for internal-external rotation, $12 \mathrm{~mm}$ for the lateral-medial displacement and $20 \mathrm{~mm}$ for the anterior-posterior displacement. The different combinations have a very limited effect on extension-flexion and proximal-distal displacement. These ranges are due in part to the higher sensitivity of the model for low weight values of the ligament constraints, as shown in Fig. 6: indeed, Fig. 7 includes combinations of both low and high ligament weights. Conversely, Table 5 shows that if at least one ligament has a high weight (1e4), maximal standard deviations remain small, their average values over the five subjects being below 1 degree and $4 \mathrm{~mm}$ respectively for knee rotations and displacements.

\section{Discussion}

In this study, a mathematical framework to include deformable ligament constraints in joint kinematic models for MBO is proposed. Previously reported in vitro experimental data $[11,26]$ are processed in order to build a specific knee kinematic model, consisting of a parallel mechanism that accurately models the joint natural motion and experimental ligament length variations (Tables 1,2). The method is applied to the knee but could be applied to other joints, such as the ankle. Indeed, ankle parallel mechanisms with isometric ligaments have already been proposed [26, 31, 32]. 
The knee parallel mechanism was presented and validated in previous studies [8-11] and it was used in a previous MBO [7]. The use of deformable ligaments as an extension of a parallel mechanism joint model for MBO was also previously presented and validated [25]. However, a full mathematical framework that could consider the three optimization methods $\left(\Delta \mathrm{L}_{0}, \Delta \mathrm{L}_{\min }, \Delta \mathrm{L}_{\theta}\right)$ was not presented. Moreover, the performance of the optimization methods in terms of ligament lengthening and contact point positions was not investigated. The present study proposes a fast and robust penalty-based method that introduces deformable ligaments in the parallel mechanism and, consequently, in the $\mathrm{MBO}$, in order to also consider the ligament length variations. The method defines quadratic constrained optimization problems that, consequently, are smooth, convex and insensitive to the initial guess. The present method can be regarded as a generalization of the previous MBO [7] with isometric (i.e., rigid) ligaments. In particular, the ligament length variations are consistent with the fixed ligament lengths of the parallel mechanism: the mechanism ligament lengths $\tilde{d}^{l}$ are very close to the mean of the experimental ligament lengths $\bar{d}^{l}(\theta)$. This method could eventually be extended to deformable contacts and to additional ligament bundles. With respect to more detailed dynamic (or quasi-static) deformable models [16, 33-39], the proposed method directly deals with kinematics rather than dynamic (or static) equilibrium, thus it requires a lower computational cost. This simplifies also personalization of the model parameters, in case a subject-specific model is required [15]: mechanical properties such as ligament stiffness and unloaded lengths are difficult to be obtained in vivo, but the method is exempted from their definition.

375 It should be noted that one of the main issues with the penalty method is the weight definition. In this study, ligament weights are chosen upon qualitative assumptions based on the literature and on the in vitro experimental data, which provided information on the level of isometry showed by the ligaments during natural motion. This indeed can be seen as a measure of the laxity of ligaments, intended as their elongation when low physiological loads are applied. However, other criteria can be used, for instance based on the mean ligament mechanical characteristics measured on in vitro studies. Moreover, some general indications could be obtained by the sensitivity analysis of the model. The sensitivity of the model to the weight variation was analyzed in this study. Results showed that the 
extension-flexion and proximal-distal displacement are not sensitive to the ligament weights, thus these DoF either are less influenced by STA or are mainly determined by the contact constraints. However, adduction-abduction, internal-external rotation, lateral-medial displacement and anteriorposterior displacement were sensitive for weights lower than 2500-3200 (when the weight for the driving constraints was 1) depending on the subject. In this sense, a weight above this range would be recommended to reduce model sensitivity. In case different weights are used between ligaments, results show that the sensitivity is reduced also if only one ligament has a weight above this range. The results also suggest that, during the considered tests, in a STA compensation perspective the adduction-abduction and internal-external rotation were mainly constrained by the ACL, the lateralmedial displacement by the MCL, and the anterior-posterior displacement by the LCL and PCL. It is worth noting that these ligaments provided the greatest constrain for the STA compensation, but they are not necessarily the greatest joint constraints for the considered motion task (i.e., the walking).

395 In the present study, the geometrical model is not personalized and the experimental ligament lengthening, used in particular for $\Delta \mathrm{L}_{\theta}$, was measured in vitro during joint natural motion. Personalization can actually improve the MBO efficiency for STA compensation [15], but the experimental procedures can be more complicated since the model parameters have to be measured on a subject, and the computational burden for model definition increases. A subject-specific model geometry can be obtained from medical imaging. For instance, ligament origins and insertions could be obtained both from static magnetic resonance and computed tomography [40]. Personalized weight bearing ligament length variations could also be obtained in vivo, but more complex protocols involving dynamic imaging techniques (such as fluoroscopy or dynamic magnetic resonance) have to be used. In this sense, $\Delta \mathrm{L}_{0}$ and $\Delta \mathrm{L}_{\text {min }}$ could be applied more easily to standard measurements, since length variation patterns are not required. However, if ligament lengthening patterns are needed, the use of the joint natural motion as a reference as done in this study could simplify the experimental protocols. Indeed, some techniques (such as dynamic magnetic resonance) have limitations in terms of field of view and velocity that can be overcome by simple tasks such as the joint natural motion. Repeatable measurements of the joint natural motion can also be easily performed in vitro, thus 
allowing definition of a reference kinematic database. Finally, promising numerical techniques have been proposed that can predict the subject-specific natural motion by standard static imaging techniques [41]. It could be noted that ligament lengthening during natural motion may represent the behavior of ligaments during gait $[23,24,42]$. Indeed, ligaments tend to remain in their isometric state, apart from lengthening due to dynamic and muscular loads that are allowed by the deformable ligaments of the model. In this sense, the ligament lengthening obtained by $\Delta \mathrm{L}_{\min }$ and $\Delta \mathrm{L}_{\theta}$ can be considered promising in vivo estimations. As for the higher computational burden, personalization could also include adjustment of the preliminary estimate of the model parameters. This is generally performed by optimization techniques, which could take from some minutes to one hour of computational time on a standard computer, depending on the specimen geometry [26]. However, these computations have to be performed only during model definition: once the personalized model is defined, each simulation takes seconds to run.

In musculoskeletal modeling, kinematic models are required, thus knee or other joint models that can represent physiological kinematics are relevant [17, 43-45]. Especially, the contact point positions are important parameters for the computation of knee contact forces [46]. Multi-body kinematic models are also particularly important when joint kinematics is estimated from skin marker measurements, to reduce errors due to the STA. The use of an anatomically accurate model of the knee based on a parallel mechanism with zero, minimized or prescribed ligament variations for STA compensation was previously validated by means of in vivo knee joint kinematics of running cycles, measured both by skin markers and by intra-cortical pins [25]. All three methods allowed reduction of the error between the model-based and the pin-measured kinematics, with respect to other techniques in the literature. $\Delta \mathrm{L}_{0}$ and $\Delta \mathrm{L}_{\theta}$ performed better on joint displacements, $\Delta \mathrm{L}_{\text {min }}$ was better for joint rotations. $\Delta \mathrm{L}_{0}$ showed higher errors on internal-external rotation, which were corrected by deformable ligaments. In the present study, application of the three methods on gait cycles confirmed these observations, and extended the analysis to ligament lengthening and contact point positions. Displacement and rotation results are comparable to data in the literature obtained by pin measurements [47-49]. Concerning the internal rotation, the pattern obtained by $\Delta \mathrm{L}_{0}$ is more distant from experimental measurements [47], 
than the patterns of $\Delta \mathrm{L}_{\min }$ and $\Delta \mathrm{L}_{\theta}$. It should be noted that the internal-external rotation of the knee is sensitive to external loads, in particular at high flexion angles [50]: deformable ligaments allow some model adaptation to the changing loading conditions that is prevented in case of isometric ligaments. Internal rotation patterns from $\Delta \mathrm{L}_{\theta}$ are the most similar to experimental measurements in this case, with a first peak at $15 \%$ and a second peak at the toe-off. As for displacements, while some studies report a high range during gait (peak about $20 \mathrm{~mm}$ for anterior displacement) [47], more recent studies based on biplane fluoroscopy measured lower displacements that are closer to the $\Delta \mathrm{L}_{0}$ and $\Delta \mathrm{L}_{\theta}$ prediction (peak about $3 \pm 2 \mathrm{~mm}$ for both anterior and medial displacements) [51]. Studies based on biplane fluoroscopy also reported in vivo contact point positions $[52,53]$ and those obtained with $\Delta \mathrm{L}_{0}$ (better than $\Delta \mathrm{L}_{\min }$ and $\Delta \mathrm{L}_{\theta}$ ) compare favourably. It is important to say that the contact points rely not only on the kinematics but also on the condyle and plateau geometries. In the proposed knee model, these geometries have been simplified to spheres and planes, which are not personalized in the present study. A subject-specific bone geometry can provide more promising results also in this case [54].

\section{Conclusions}

A multi-body optimization framework is presented to introduce deformable ligaments and articular contacts in a kinematic knee model, for soft-tissue artifact compensation in gait analysis at the lower limb. Two penalty-based methods (featuring minimized and prescribed ligament length variations respectively) are implemented as an extension of a previous method based on Lagrange multipliers and featuring isometric ligaments. The mathematical framework proved to be robust and fast. Moreover, it is based on purely kinematic assumptions that simplifies computations and model definition for in vivo measurements. Although the knee model was not personalized in this study, the multi-body framework allows implementation of both a subject-specific and a general model geometry.

The methods were applied to the analysis of the gait cycle of five subjects. The results show that all three methods make it possible to obtain kinematic patterns for knee rotations and displacements that are consistent with measurements performed in vivo by bone-pins or biplane fluoroscopy. The 
methods with deformable ligaments allowed some model adaptation to take into account the effect of

loads on the tibiofemoral motion, particularly evident for the knee internal rotation, ligament lengthening and contact point positions. In general, the results from the zero and prescribed ligament length variation methods were better for joint displacements, while the minimized ligament length variation method obtained rotation patterns closer to results from the literature. A sensitivity analysis showed that the model sensitivity to the variations of the weights of the penalty-based methods could be reduced by setting these weights above a certain range.

As far as a knee kinematic model is used, particularly in musculoskeletal modeling, the proposed multi-body methods seem a good compromise between too simple non-physiological kinematic models such as the hinge and too complex deformable models based on the solution of the static or dynamic equilibrium.

\section{References}

1. Leardini A, Chiari L, Della Croce U, Cappozzo A (2005) Human movement analysis using stereophotogrammetry. Part 3. Soft tissue artifact assessment and compensation. Gait Posture $21: 212-225$

2. Akbarshahi M, Schache AG, Fernandez JW, Baker R, Banks S, Pandy MG (2010) Non-invasive assessment of soft-tissue artifact and its effect on knee joint kinematics during functional activity. J Biomech 43:1292-1301

3. Peters A, Galna B, Sangeux M, Morris M, Baker R (2010) Quantification of soft tissue artifact in lower limb human motion analysis: a systematic review. Gait Posture 31:1-8

4. Lu TW, O’Connor JJ (1999) Bone position estimation from skin marker co-ordinates using global optimisation with joint constraints. J Biomech 32:129-134

5. Reinbolt JA, Schutte JF, Fregly BJ, Koh BI, Haftka RT, George AD, Mitchell KH (2005) Determination of patient-specific multi-joint kinematic models through two-level optimization. $\mathrm{J}$ Biomech 38:621-626

6. Andersen MS, Damsgaard M, Rasmussen J (2009) Kinematic analysis of over-determinate biomechanical systems. Comput Methods Biome 12:371-384

7. Duprey S, Chèze L, Dumas R (2010) Influence of joint constraints on lower limb kinematics estimation from skin markers using global optimization. J Biomech 43:2858-2862

8. Wilson D, Feikes J, O’Connor J (1998) Ligaments and articular contact guide passive knee flexion. J Biomech 31:1127:1136 
9. Parenti-Castelli V, Di Gregorio R (2000) Parallel mechanisms applied to the human knee passive motion simulation. In: Advances in Robot Kinematics. Springer Netherlands, pp 333-344

10. Feikes JD, O'Connor JJ, Zavatsky AB (2003) A constraint-based approach to modelling the mobility of the human knee joint. J Biomech 36:125-129

11. Ottoboni A, Parenti-Castelli V, Sancisi N, Belvedere C, Leardini A (2010) Articular surface approximation in equivalent spatial parallel mechanism models of the human knee joint: an experiment-based assessment. P I Mech Eng H 224:1121-1132

12. Sancisi N, Parenti-Castelli V (2011) A new kinematic model of the passive motion of the knee inclusive of the patella. J Mech Robot 3:041003.

13. Menschik A (1974) Mechanik des Kniegelenks, Teil 1. Z Orthop 112:481-495

14. O’Connor JJ, Lu TW, Wilson DW, Feikes JD, Leardini A (1998) Review: Diarthrodial jointskinematic pairs, mechanisms or flexible structures? Comput Methods Biome 1:123-150

15. Clément J, Dumas R, Hagemeister N, de Guise JA (2015) Soft tissue artifact compensation in knee kinematics by multi-body optimization: Performance of subject-specific knee joint models. $\mathrm{J}$ Biomech 48:3796-3802

16. Sancisi N, Parenti-Castelli V (2011) A sequentially-defined stiffness model of the knee. Mech Mach Theory 46:1920-1928

17. Dumas R, Moissenet F, Gasparutto X, Chèze L (2012) Influence of joint models on lower-limb musculo-tendon forces and three-dimensional joint reaction forces during gait. P I Mech Eng $\mathrm{H}$ 226:146-160

18. Moissenet F, Chèze L, Dumas R (2012) Anatomical kinematic constraints: consequences on musculo-tendon forces and joint reactions. Multibody Syst Dyn 28:125-141

19. Moissenet F, Chèze L, Dumas R (2014) A 3D lower limb musculoskeletal model for simultaneous estimation of musculo-tendon, joint contact, ligament and bone forces during gait. J Biomech 47:50-58

20. Rovick JS, Reuben JD, Schrager RJ, Walker PS (1991) Relation between knee motion and ligament length patterns. Clin Biomech 6:213-220

21. Hsieh YF, Draganich LF (1997) Knee kinematics and ligament lengths during physiologic levels of isometric quadriceps loads. The Knee 4:145-154

22. Bergamini E, Pillet H, Hausselle J, Thoreux P, Guerard S, Camomilla V, Cappozzo A, Skalli W (2011) Tibio-femoral joint constraints for bone pose estimation during movement using multibody optimization. Gait Posture 33:706-711

23. Liu F, Gadikota HR, Kozánek M, Hosseini A, Yue B, Gill TJ, Rubash HE, Li G (2011) In vivo length patterns of the medial collateral ligament during the stance phase of gait. Knee Surg Sport Tr A19:719-727 
25. Gasparutto X, Sancisi N, Jacquelin E, Parenti-Castelli V, Dumas R (2015) Validation of a multibody optimization with knee kinematic models including ligament constraints. J Biomech 48:1141-1146

26. Parenti-Castelli V, Sancisi N (2013) Synthesis of spatial mechanisms to model human joints. In: 21 st Century Kinematics. Springer London, pp 49-84

27. Wu G, Siegler S, Allard P, Kirtley C, Leardini A, Rosenbaum D, Whittle M, D'Lima D, Cristofolini L, Witte H, Schmid O, Stokes I (2002) ISB recommendation on definitions of joint coordinate system of various joints for the reporting of human joint motion-part I: ankle, hip, and spine. J Biomech 35:543-548

28. Sancisi N, Parenti-Castelli V (2011) On the role of ligaments in the guidance of the human knee passive motion. In: Proceedings of Euromech Colloquium 511, pp 1-9

29. De Jalon JG, Unda J, Avello A (1986) Natural coordinates for the computer analysis of multibody systems. Comput Method Appl M 56:309-327

30. Dumas R, Chèze L (2007) 3D inverse dynamics in non-orthonormal segment coordinate system. Med Biol Eng Comput 45:315-322

31. Franci R, Parenti-Castelli V, Belvedere C, Leardini A (2009) A new one-DOF fully parallel mechanism for modelling passive motion at the human tibiotalar joint. J Biomech 42:1403-1408

32. Sancisi N, Baldisserri B, Parenti-Castelli V, Belvedere C, Leardini A (2014) One-degree-offreedom spherical model for the passive motion of the human ankle joint. Med Biol Eng Comput $52: 363-373$

33. Wismans J, Veldpaus F, Janssen J (1980) A three-dimensional mathematical model of the knee joint. J Biomech 13:677-385

34. Blankevoort L, Huiskes R (1996) Validation of a three-dimensional model of the knee. J Biomech 29: 955-961

35. Bei Y, Fregly B (2004) Multibody dynamic simulation of knee contact mechanics. Med Eng Phys 26: 777-789

36. Caruntu DI, Hefzy MS (2004) 3-D anatomically based dynamic modeling of the human knee to include tibio-femoral and patello-femoral joints. J Biomech Eng 126:44-53

37. Shelburne KB, Pandy MG, Anderson FC, Torry MR (2004) Pattern of anterior cruciate ligament force in normal walking. J Biomech 37:797-805

38. Guess TM (2012) Forward dynamics simulation using a natural knee with menisci in the multibody framework. Multibody Syst Dyn 28:37-53

39. Lenhart RL, Kaiser J, Smith CR, Thelen DG (2015) Prediction and validation of load-dependent behavior of the tibiofemoral and patellofemoral joints during movement. Ann Biomed Eng 43:2675-2685 
40. Ascani D, Mazzà C, De Lollis A, Bernardoni M, Viceconti M (2015) A procedure to estimate the origins and the insertions of the knee ligaments from computed tomography images. J Biomech 48:233-237

41. Sancisi N, Conconi M, Parenti-Castelli V (2015) Prediction of the subject-specific knee passive motion from non-invasive measurements. In: Proceedings of ISB 2015, pp 1-2

42. Wu JL, Hosseini A, Kozanek M, Gadikota HR, Gill TJ, Li G (2010) Kinematics of the anterior cruciate ligament during gait. Am J Sport Med 38:1475-1482

43. Seth A, Sherman M, Eastman P, Delp S (2010) Minimal formulation of joint motion for biomechanisms. Nonlinear Dynam 62: 291-303

44. Ribeiro A, Rasmussen J, Flores P, Silva LF (2011) Modeling of the condyle elements within a biomechanical knee model. Multibody Syst Dyn 28:181-197

45. Hu C, Lu T, Chen S (2013) Influence of model complexity and problem formulation on the forces in the knee calculated using optimization methods. Biomed Eng Online 7:12-20

46. Winby CR, Lloyd DG, Besier TF, Kirk TB (2009) Muscle and external load contribution to knee joint contact loads during normal gait. JBiomech 42:2294-2300

47. Lafortune MA, Cavanagh PR, Sommer III HJ, Kalenak A (1992) Three-dimensional kinematics of the human knee during walking. J Biomech 25:347-357

48. Reinschmidt C, Van Den Bogert AJ, Lundberg A, Nigg BM, Murphy N, Stacoff A, Stano A (1997) Tibiofemoral and tibiocalcaneal motion during walking: external vs. skeletal markers. Gait Posture 6:98-109

49. Benoit DL, Ramsey DK, Lamontagne MA, Xu L, Wretenberg P, Renström P (2007) In vivo knee kinematics during gait reveals new rotation profiles and smaller translations. Clin Orthop Relat $\mathrm{R}$ 454:81-88

50. Blankevoort L, Huiskes R, De Lange A (1988) The envelope of passive knee joint motion. J Biomech 21:705-720

51. Myers CA, Torry MR, Shelburne KB, Giphart JE, LaPrade RF, Woo S-L, Steadman JR (2012) In vivo tibiofemoral kinematics during 4 functional tasks of increasing demand using biplane fluoroscopy. Am J Sport Med 40:170-178

52. Li G, DeFrate LE, Park SE, Gill TJ, Rubash HE (2005) In vivo articular cartilage contact kinematics of the knee: an investigation using dual-orthogonal fluoroscopy and magnetic resonance image-based computer models. Am J Sport Med 33:102-107

53. Farrokhi S, Voycheck CA, Klatt BA, Gustafson JA, Tashman S, Fitzgerald GK (2014) Altered tibiofemoral joint contact mechanics and kinematics in patients with knee osteoarthritis and episodic complaints of joint instability. Clin Biomech 29:629-635

54. Clément J, Cresson T, Hagemeister N, Dumas R, de Guise JA (2015) Estimating joint space of the knee during weight-bearing squatting activity using motion capture - preliminary results of a new method. Comput Method Biomec 18:1910-1901 


\section{Figure captions}

605 Figure 1 : Kinematic model of the knee: isometric ligaments and contact surface approximations are shown together with the virtual markers representing the kinematic constraints.

Figure 2 : Knee joint angles obtained by $\Delta \mathrm{L}_{0}, \Delta \mathrm{L}_{\text {min }}, \Delta \mathrm{L}_{\theta}$ as a function of gait cycle: mean $\pm 1 \mathrm{SD}$ over the five analyzed subjects.

Figure 3: Knee joint displacements obtained by $\Delta \mathrm{L}_{0}, \Delta \mathrm{L}_{\min }, \Delta \mathrm{L}_{\theta}$ as a function of gait cycle: mean $\pm 1 \mathrm{SD}$ over the five analyzed subjects.

Figure 4: Ligament lengthening obtained by $\Delta \mathrm{L}_{0}, \Delta \mathrm{L}_{\min }, \Delta \mathrm{L}_{\theta}$ as a function of gait cycle: mean $\pm 1 \mathrm{SD}$ over the five analyzed subjects.

Figure 5: Medial and lateral contact point positions obtained by $\Delta \mathrm{L}_{0}, \Delta \mathrm{L}_{\min }, \Delta \mathrm{L}_{\theta}$ on the axial plane: mean \pm 1 SD over the five analyzed subjects. Colors represent $\%$ of gait cycle: black $=0 \%$, yellow $=100 \%$.

Figure 6: Knee kinematics obtained by changing the weights between the driving and the ligament constraints on a representative subject. Colors represent the different ligament weights: green $=$ 1 , blue $=2 \mathrm{e} 4$, red $=1 \mathrm{e} 4$ (chosen in the model).

Figure 7: Knee kinematics obtained by changing the relative weights between the ligament constraints on the same subject as in Fig. 6. All considered combinations are in blue; the combination used in the model is in red.

Figure 8: Ligament lengthening obtained by changing the weights between the driving and the ligament constraints on the same subject as Fig. 6. Colors represent the different ligament weights: green $=1$, blue $=2 \mathrm{e} 4$, red $=1 \mathrm{e} 4($ chosen in the model).

625 Figure 9: Ligament lengthening obtained by changing the relative weights between the ligament constraints on the same subject as in Fig. 6. All considered combinations are in blue; the combination used in the model is in red.

\section{Table captions}

Table 1: Knee model geometrical parameters and corresponding virtual markers.

Table 2: Coefficients $a^{l}$ for the polynomial interpolation of the experimental ligament lengths.

Table 3: Ligament weights in the penalty-based methods.

Table 4: Root mean square (RMS) differences between the model and the experimental ligament lengths measured in vitro (expressed as \% value of the ligament length at the neutral pose $d^{l}(0)$ ).

Table 5: Sensitivity of the model to relative ligament weights: mean maximal standard deviations associated to each group. 
Figure 1

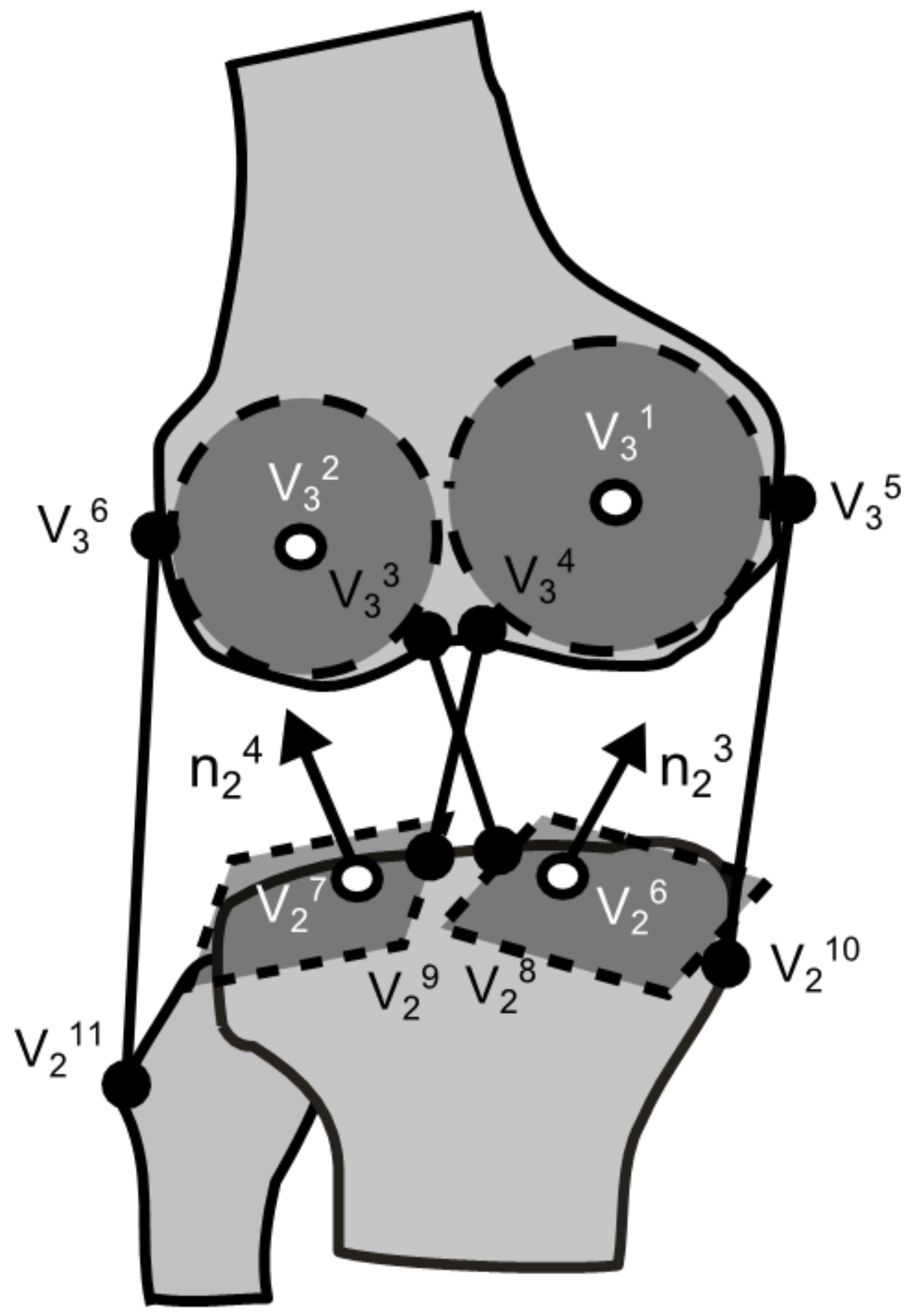


Figure 2
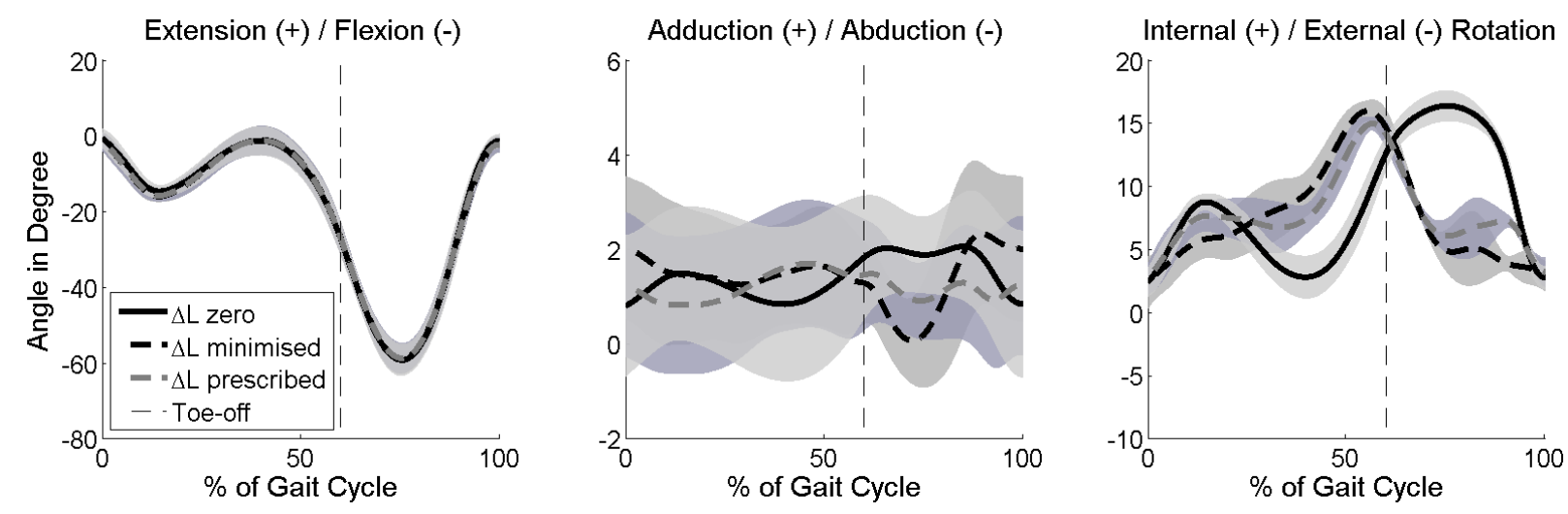
Figure 3
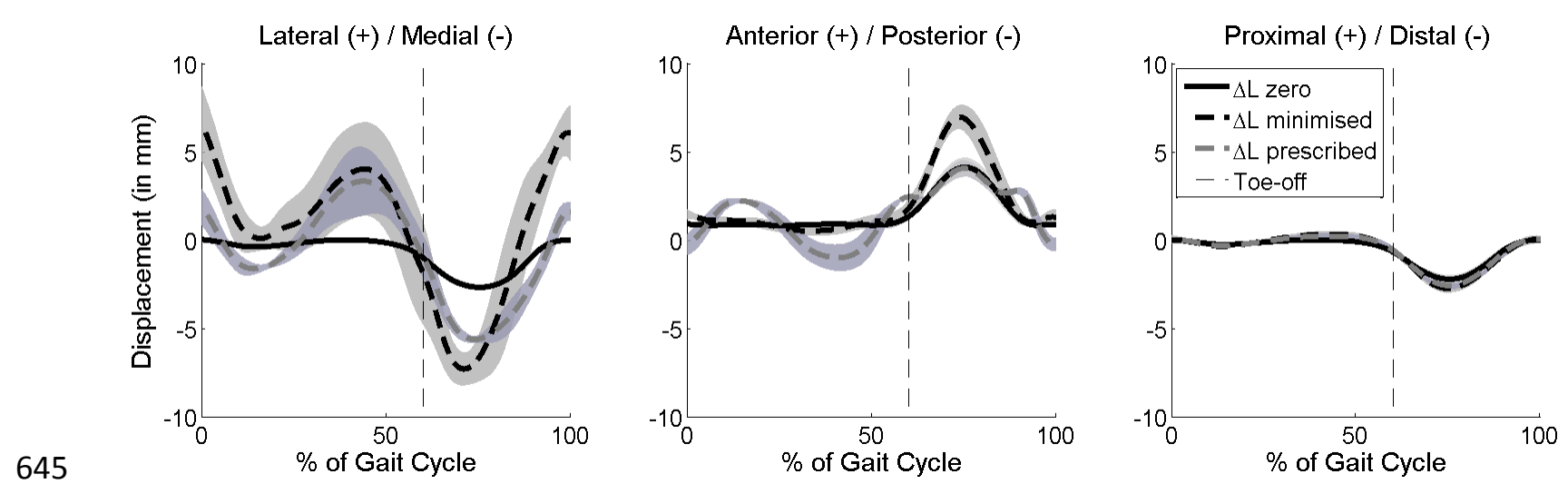
Figure 4
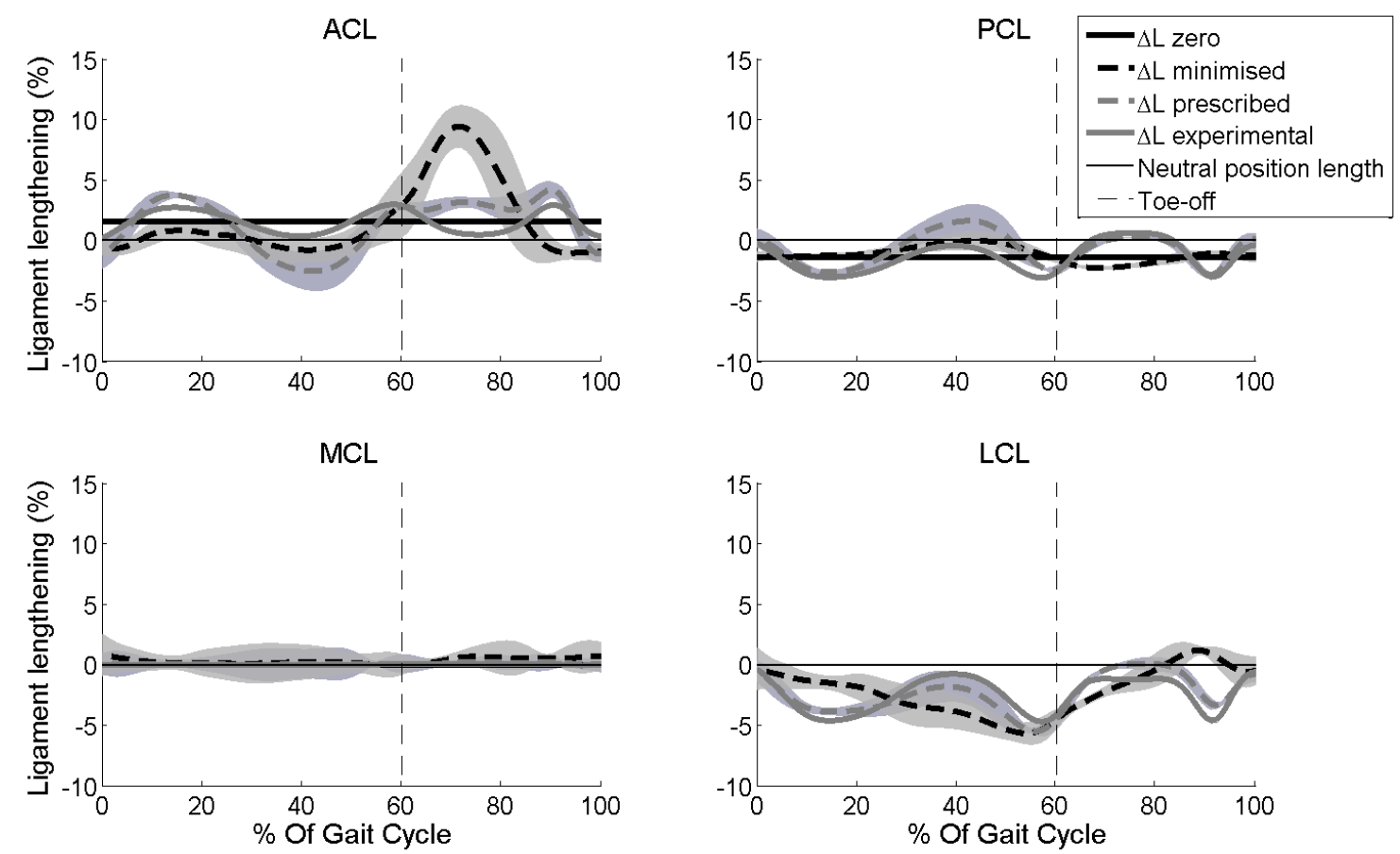
Figure 5
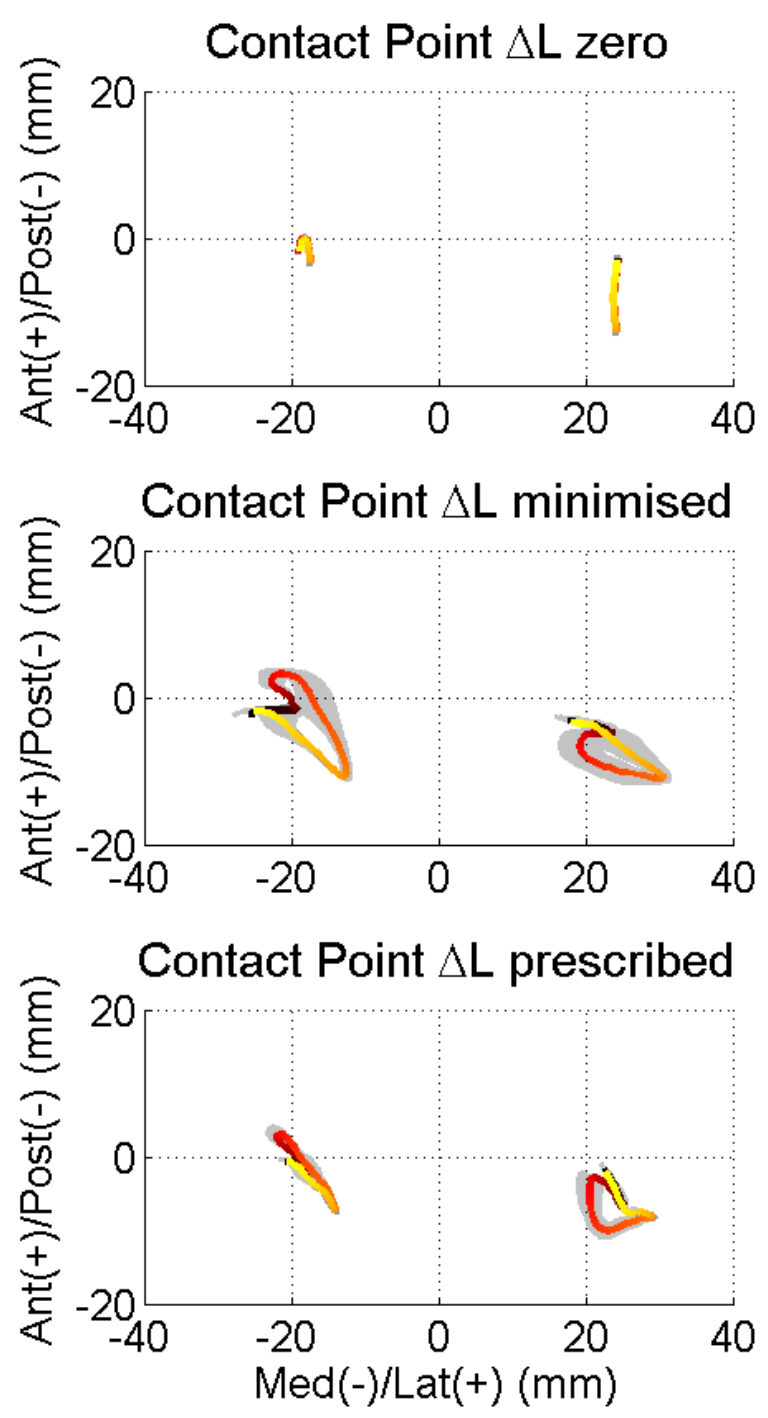
Figure 6
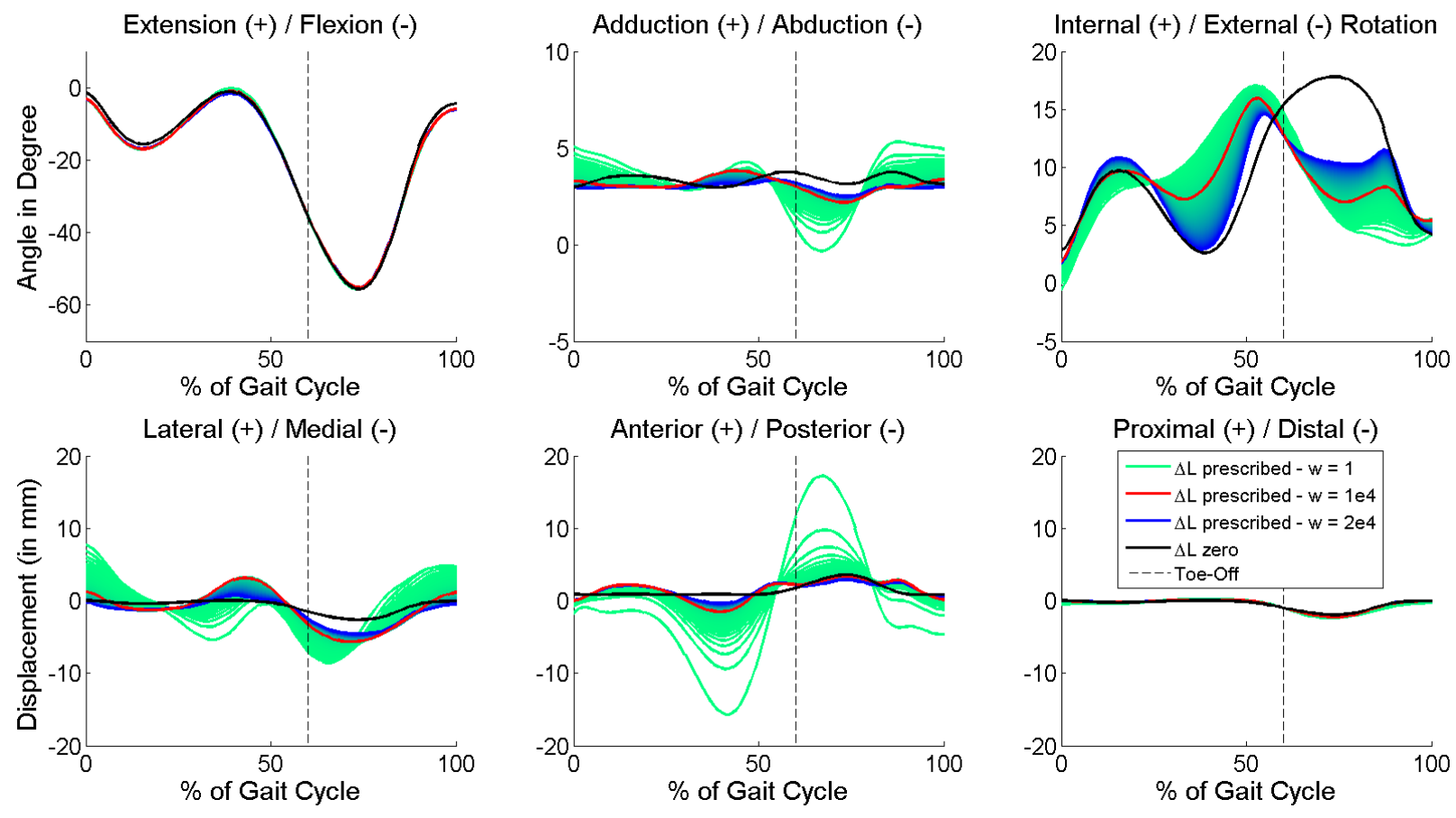
Figure 7
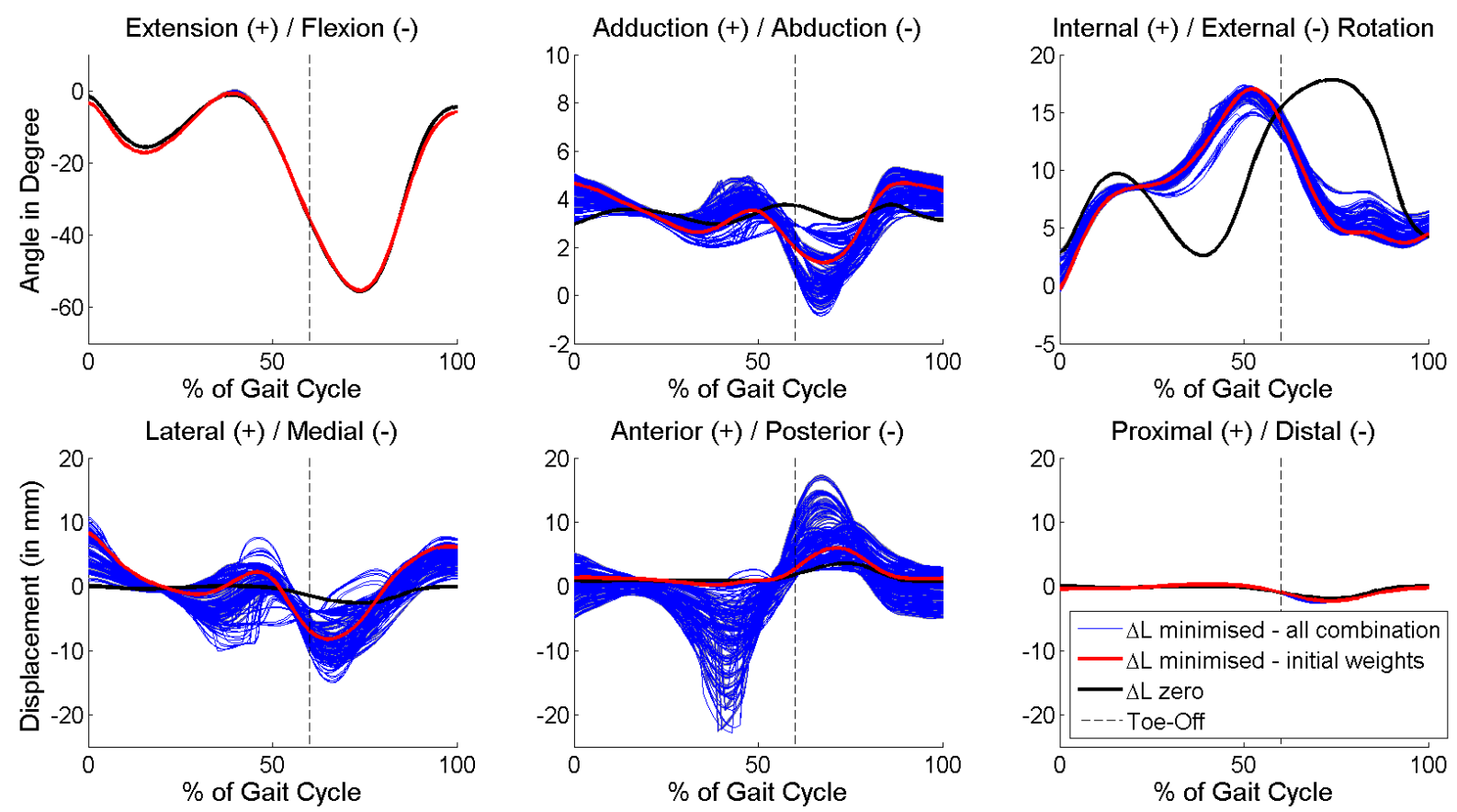
Figure 8
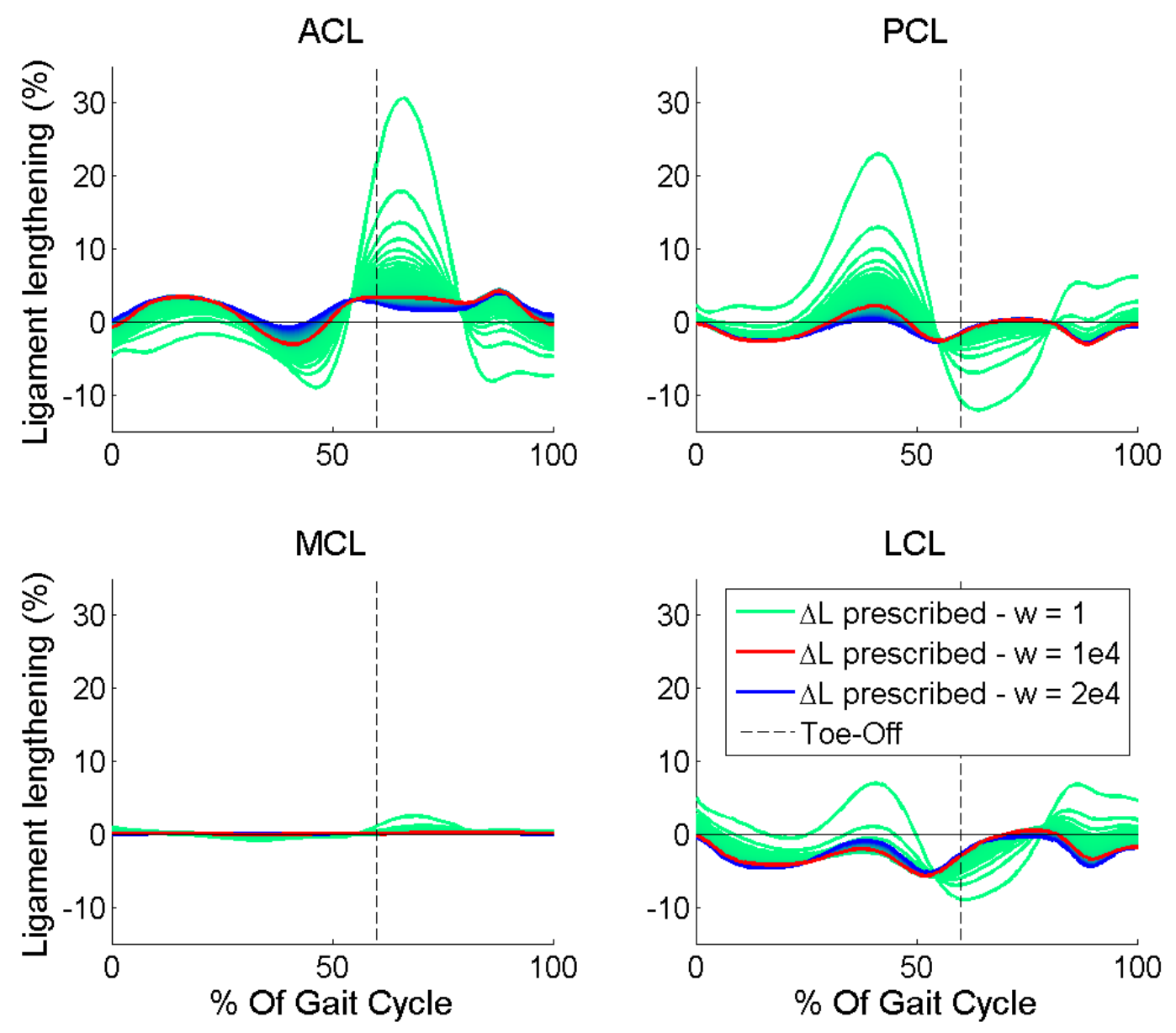
Figure 9
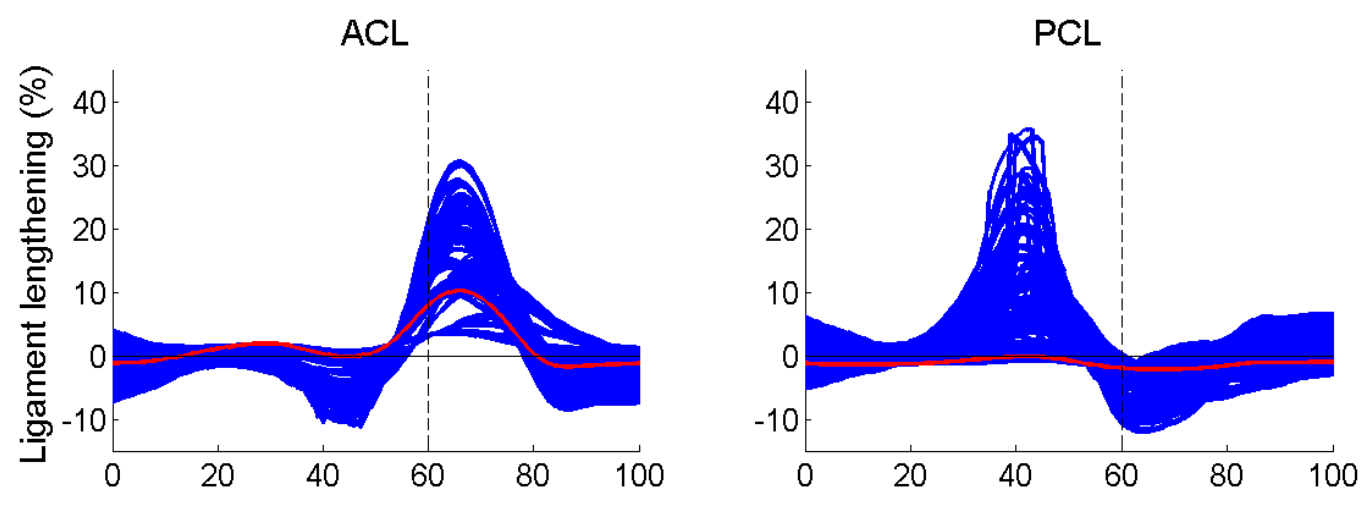

MCL

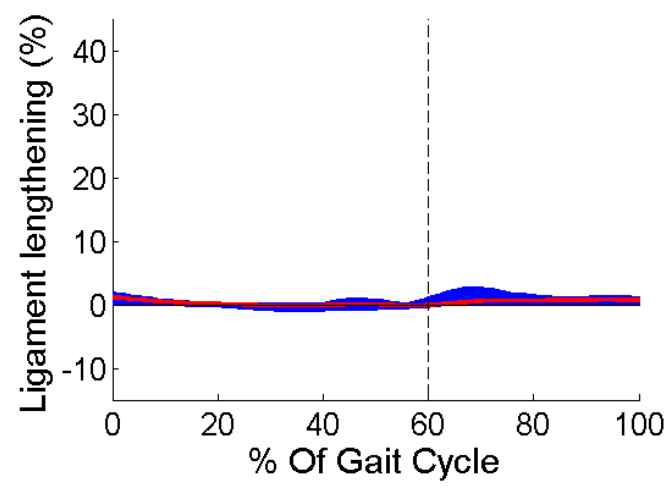

LCL

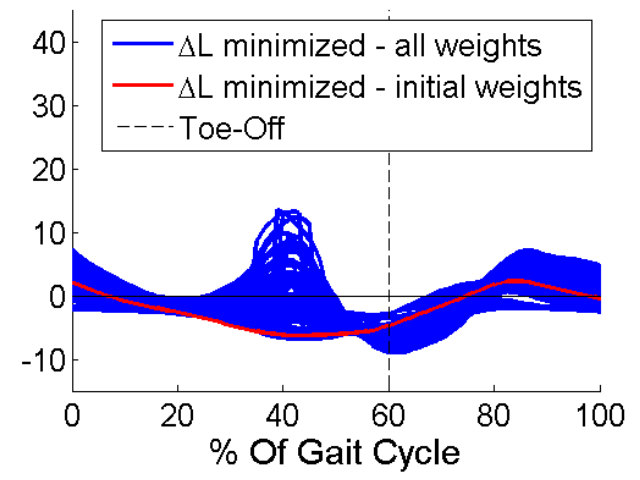




\begin{tabular}{|c|c|c|c|c|c|}
\hline \multirow{2}{*}{ Segment } & \multirow{2}{*}{$\begin{array}{l}\text { Virtual } \\
\text { marker }\end{array}$} & \multirow{2}{*}{$\begin{array}{l}\text { Anatomical point } \\
\text { or orientation } \\
\text { vector }\end{array}$} & \multicolumn{3}{|c|}{$\begin{array}{c}\begin{array}{c}\text { Coordinates }(\mathrm{mm}) \text { or components in } \\
\text { femur/tibia SCSs }\end{array} \\
\end{array}$} \\
\hline & & & $\mathbf{X}$ & $\mathbf{Y}$ & $\mathbf{Z}$ \\
\hline \multirow[t]{6}{*}{ Femur } & $V_{3}^{1}$ & Medial condyle center & 0.2458 & 3.4071 & -23.2019 \\
\hline & $V_{3}^{2}$ & Lateral condyle center & -3.2853 & 2.1225 & 26.2054 \\
\hline & $V_{3}^{3}$ & ACL origin & -6.7712 & 7.5255 & 9.1575 \\
\hline & $V_{3}^{4}$ & PCL origin & -2.6610 & -1.0906 & -2.1857 \\
\hline & $V_{3}^{5}$ & MCL origin & 2.7608 & 5.7798 & -47.6279 \\
\hline & $V_{3}^{6}$ & LCL origin & 3.2800 & 2.2812 & 36.1895 \\
\hline \multirow[t]{8}{*}{ Tibia } & $V_{2}^{6}$ & Medial tibial plateau & -2.1344 & -28.6241 & -19.1308 \\
\hline & $V_{2}^{7}$ & Lateral tibial plateau & -2.7946 & -26.0861 & 24.3679 \\
\hline & $n_{2}^{3}$ & Medial normal & 0.0675 & 0.9896 & -0.1273 \\
\hline & $n_{2}^{4}$ & Lateral normal & -0.0881 & 0.9942 & 0.0617 \\
\hline & $V_{2}^{8}$ & ACL insertion & 12.7709 & -26.1454 & -0.9269 \\
\hline & $V_{2}^{9}$ & PCL insertion & -25.8519 & -38.1449 & -3.5321 \\
\hline & $V_{2}^{10}$ & MCL insertion & 2.1345 & -117.0682 & -5.7872 \\
\hline & $V_{2}^{11}$ & LCL insertion & -24.2639 & -47.9992 & 37.1213 \\
\hline
\end{tabular}

The numbering of the virtual markers follows [7]. The ligament lengths at the neutral pose $d^{l}(0)(l=$ $3,4,5,6)$ can be computed from the distances between origin and the insertion of each ligament, while 670 the mechanism optimal ligament lengths used in $\Delta \mathrm{L}_{0}$ are $\tilde{d}^{3}=40.53 \mathrm{~mm}, \tilde{d}^{4}=43.26 \mathrm{~mm}, \tilde{d}^{5}=$ $129.70 \mathrm{~mm}$. The model sphere radii are $\tilde{d}^{1}=32.32 \mathrm{~mm}, \tilde{d}^{2}=28.34 \mathrm{~mm}$. The mean ligament length used for the model $\Delta \mathrm{L}_{\min }$ were not constant among subject, there mean value where $\bar{d}^{3}=40.62 \pm$ $0.11 \mathrm{~mm}, \bar{d}^{4}=43.12 \pm 0.13 \mathrm{~mm}, \bar{d}^{5}=129.80 \pm 0.05 \mathrm{~mm}, \bar{d}^{6}=55.94 \pm 0.23 \mathrm{~mm}$. 
675 Table 2

\begin{tabular}{llll}
\hline ACL $(l=3)$ & PCL $(l=4)$ & MCL $(l=5)$ & LCL $(l=6)$
\end{tabular}

\begin{tabular}{lllll}
\hline$a_{1}^{l}$ & $-2.5 \mathrm{e}-3$ & $2.7 \mathrm{e}-3$ & $2.3336 \mathrm{e}-4$ & $3.5 \mathrm{e}-3$ \\
$a_{2}^{l}$ & $-1.4023 \mathrm{e}-5$ & $-4.2080 \mathrm{e}-5$ & $3.2597 \mathrm{e}-5$ & $-1.5564 \mathrm{e}-4$ \\
$a_{3}^{l}$ & $3.2187 \mathrm{e}-6$ & $-8.5132 \mathrm{e}-6$ & $1.3686 \mathrm{e}-6$ & $-1.9254 \mathrm{e}-5$ \\
$a_{4}^{l}$ & $9.1037 \mathrm{e}-8$ & $-2.4381 \mathrm{e}-7$ & $2.4415 \mathrm{e}-8$ & $-5.7922 \mathrm{e}-7$ \\
$a_{5}^{l}$ & $1.0491 \mathrm{e}-9$ & $-3.0408 \mathrm{e}-9$ & $1.7782 \mathrm{e}-10$ & $-7.9173 \mathrm{e}-9$ \\
$a_{6}^{l}$ & $5.8532 \mathrm{e}-12$ & $-1.8055 \mathrm{e}-11$ & $2.2257 \mathrm{e}-13$ & $-5.1875 \mathrm{e}-11$ \\
$a_{7}^{l}$ & $1.3559 \mathrm{e}-14$ & $-4.1819 \mathrm{e}-14$ & $-1.7572 \mathrm{e}-15$ & $-1.3239 \mathrm{e}-13$ \\
\hline
\end{tabular}


Table 3

$$
\operatorname{ACL}(l=3) \quad \text { PCL }(l=4) \quad \text { MCL }(l=5) \quad \text { LCL }(l=6)
$$

\begin{tabular}{lcccc}
\hline$\Delta \mathbf{L}$ minimized & $1 \mathrm{e} 3$ & $1 \mathrm{e} 4$ & $1 \mathrm{e} 2$ & $1 \mathrm{e} 0$ \\
$\Delta \mathbf{L}$ prescribed & $1 \mathrm{e} 4$ & $1 \mathrm{e} 4$ & $1 \mathrm{e} 4$ & $1 \mathrm{e} 4$ \\
\hline
\end{tabular}

680 
Table 4

RMS difference (\%)

$\Delta \mathrm{L}$ zero

$\Delta \mathrm{L}$ minimised

$\Delta \mathrm{L}$ prescribed

\begin{tabular}{lccc}
\hline ACL & 1.7 & 2.9 & 1.7 \\
PCL & 1.8 & 0.7 & 1.1 \\
MCL & 0.05 & 0.3 & 0.1 \\
LCL & - & 2.1 & 0.9 \\
\hline
\end{tabular}


Rotations (deg)

Displacements (mm)

\begin{tabular}{|c|c|c|c|c|c|c|}
\hline Group & $\begin{array}{l}\text { Extension (+) } \\
\text { Flexion (-) }\end{array}$ & $\begin{array}{c}\text { Adduction (+) } \\
\text { Abduction(-) }\end{array}$ & $\begin{array}{l}\text { Internal (+) } \\
\text { External (-) }\end{array}$ & $\begin{array}{l}\text { Lateral (+) } \\
\text { Medial (-) }\end{array}$ & $\begin{array}{l}\text { Anterior (+) } \\
\text { Posterior (-) }\end{array}$ & $\begin{array}{c}\text { Proximal (+) } \\
\text { Distal (-) }\end{array}$ \\
\hline ACL & 0.1 & 0.3 & 0.4 & 2.5 & 3.4 & 0 \\
\hline PCL & 0.1 & 0.9 & 0.6 & 2.8 & 1.8 & 0.3 \\
\hline MCL & 0.1 & 0.5 & 0.7 & 1.5 & 3.6 & 0.1 \\
\hline LCL & 0.1 & 0.6 & 0.6 & 2.9 & 1.5 & 0.2 \\
\hline
\end{tabular}

